PROCEEDINGS OF THE AMERICAN MATHEMATICAL SOCIETY

Volume 124, Number 11, November 1996

\title{
BOUNDED EIGENFUNCTIONS AND ABSOLUTELY CONTINUOUS SPECTRA FOR ONE-DIMENSIONAL SCHRÖDINGER OPERATORS
}

\author{
BARRY SIMON
}

(Communicated by Palle E. T. Jorgensen)

\begin{abstract}
We provide a short proof of that case of the Gilbert-Pearson theorem that is most often used: That all eigenfunctions bounded implies purely a.c. spectrum. Two appendices illuminate Weidmann's result that potentials of bounded variation have strictly a.c. spectrum on a half-axis.
\end{abstract}

\section{§1. IntRoduCtion AND REDUCTION TO $m$-FUnCTIONS}

In this note, I want to consider Schrödinger operators and Jacobi matrices on a half-line. Specifically, we'll consider the operator $h$ on $\ell^{2}\left(\mathbb{Z}_{+}\right)$(with $\mathbb{Z}_{+}=$ $\{1,2, \ldots\})$ given by

$$
\begin{aligned}
(h u)(n) & =u(n+1)+u(n-1)+v(n) u(n), \\
u(0) & =0,
\end{aligned}
$$

and the self-adjoint operator on $L^{2}(0, \infty)$

$$
\begin{aligned}
(H u)(x) & =-u^{\prime \prime}(x)+V(x) u(x), \\
u(0) & =0,
\end{aligned}
$$

where we suppose

$$
\Gamma(V) \equiv \sup _{x}\left(\int_{x-1}^{x+1}|V(y)|^{2}\right)<\infty .
$$

For any $E \in \mathbb{C}$, define two solutions $u_{1}, u_{2}$ of the formal difference (resp. differential) equation $h u=E u$ (resp. $H u=E u$ ) with boundary conditions:

$$
\begin{array}{ll}
u_{1}(0, E)=0, & u_{1}(1, E)=1, \\
u_{2}(0, E)=1, & u_{2}(1, E)=0,
\end{array}
$$

in the discrete case and

$$
\begin{array}{ll}
u_{1}(0, E)=0, & u_{1}^{\prime}(0, E)=1, \\
u_{2}(0, E)=1, & u_{2}^{\prime}(0, E)=0,
\end{array}
$$

Received by the editors April 3, 1995 and, in revised form, April 24, 1995.

1991 Mathematics Subject Classification. Primary 34L40.

This material is based upon work supported by the National Science Foundation under Grant No. DMS-9401491. The Government has certain rights in this material. 
in the continuous case.

Let $S=\left\{E \in \mathbb{R} \mid u_{1}\right.$ and $u_{2}$ are bounded on $\left.[0, \infty)\right\}$. Then our purpose here is to prove

Theorem 1. On $S$, the spectral measure $\rho$ for $h$ (resp. $H)$ is purely absolutely continuous in the sense that

(i) $\rho_{\mathrm{ac}}(T)>0$ for any $T \subset S$ with $|T|>0$ (where $|\cdot|=$ Lebesgue measure).

(ii) $\rho_{\text {sing }}(S)=0$.

This theorem is not new. In [9], [8], [11], [14], Gilbert, Kahn, and Pearson proved a complete characterization of the essential support of $\rho_{\mathrm{ac}}$ in terms of mutually subordinate solutions. Their approach has the advantage of not requiring (1.3). Behncke [2] and Stolz [17] have noted that $V$ uniformly $L_{\text {loc }}^{1}$ with bounded eigenfunctions allows one to use the Gilbert-Pearson theory. Virtually all applications of [9], [11] use the weaker Theorem 1. There seems to be some point in the short proof I'll present here which avoids some of their tricky calculations and which makes the result transparent. In addition, we'll obtain explicit bounds on $m$-functions.

I should mention earlier work of Carmona [4] (which is weaker than Theorem 1) and related work of Briet-Mourre [3].

As with Gilbert-Pearson, our proof uses the theory of Weyl $m$-functions. For $E \in \mathbb{C}_{+}=\{z \mid \operatorname{Im} z>0\}$, we can find a unique solution $u_{+}(n, E)\left(\right.$ resp. $\left.u_{+}(x, E)\right)$ of $(1.1 \mathrm{a}) /(1.2 \mathrm{a})$ with $u_{+} \in \ell^{2}$ (resp. $\left.L^{2}\right)$ at infinity, normalized by

$$
u_{+}(0, E)=1 \text {. }
$$

Then one defines the $m$ function by

$$
m_{+}(E)=u_{+}(1, E)
$$

in the discrete case and

$$
m_{+}(E)=u_{+}^{\prime}(0, E)
$$

in the continuous case.

By looking at the Wronskian of $u_{+}$and $\bar{u}_{+}$, one gets the well-known formula:

$$
\operatorname{Im} m_{+}(E)=\operatorname{Im} E \sum_{n=1}^{\infty}\left|u_{+}(n, E)\right|^{2}
$$

in the discrete case and

$$
\operatorname{Im} m_{+}(E)=\operatorname{Im} E \int_{0}^{\infty}\left|u_{+}(x, E)\right|^{2} d x
$$

in the continuous case.

It is known (see [5], [16]) that

$$
d \rho(E)=\frac{1}{\pi} \lim _{\epsilon \downarrow 0} \operatorname{Im} m_{+}(E+i \epsilon) d E .
$$

It follows [1], [7] by the de la Vallée-Poussin theorem that

$$
\rho_{\text {sing }} \text { is supported on }\left\{E \mid \lim _{\epsilon \downarrow 0} \operatorname{Im} m_{+}(E+i \epsilon)=\infty\right\}
$$


and

$$
d \rho_{\mathrm{ac}}(E)=\frac{1}{\pi} \operatorname{Im} m_{+}(E+i 0) d E .
$$

Thus, Theorem 1 is an immediate consequence of

Theorem 2. If $E \in S$, then

$$
\begin{aligned}
& \text { (i) } \underline{\lim } \operatorname{Im} m_{+}(E+i 0)>0 \text {, } \\
& \text { (ii) } \quad \overline{\lim }\left|m_{+}(E+i 0)\right|<\infty \text {. }
\end{aligned}
$$

Remark. While the results are stated for the half-line with Dirichlet boundary conditions, Theorem 2 immediately implies the result for any fixed boundary condition and for the whole line. For it is known [1], [16] that the essential support $d \rho_{\mathrm{ac}, \theta}$ for $\theta$ boundary conditions (given by $\sin (\theta) u^{\prime}(0)+\cos (\theta) u(0)=0$ ) is $\theta$ independent and that $d \rho_{\text {sing, } \theta}$ is supported on the set where $m_{+}(E+i 0)=-\cot (\theta)$, which cannot happen if $(1.10) /(1.11)$ holds. For the whole line, we can define $S$ via the right half-line condition from which $(1.10) /(1.11)$ and the formulas (for the continuous case; the discrete case is similar)

$$
\begin{aligned}
& d \rho_{1}(E)=-\lim _{\epsilon \downarrow 0} \frac{1}{\pi} \operatorname{Im}\left(\frac{1}{m_{+}(E+i \epsilon)+m_{-}(E+i \epsilon)}\right) d E, \\
& d \rho_{2}(E)=\lim _{\epsilon \downarrow 0} \frac{1}{\pi} \operatorname{Im}\left(\frac{1}{m_{+}(E+i 0)^{-1}+m_{-}(E+i 0)^{-1}}\right) d E
\end{aligned}
$$

imply $\rho_{i, \operatorname{sing}}(S)=0$.

It is a pleasure to thank F. Gesztesy, A. Kiselev, and G. Stolz for useful discussions.

\section{$\S 2$. The Jacobi matrix CASE}

In this section, we'll prove Theorem 2 in the discrete case. Define the fundamental or transfer matrix by

$$
T(E, n, 0)=\left(\begin{array}{cc}
u_{1}(n+1, E) & u_{2}(n+1, E) \\
u_{1}(n, E) & u_{2}(n, E)
\end{array}\right)
$$

and then

$$
T(E, n, m)=T(E, n, 0) T(E, m, 0)^{-1} .
$$

$T$ is defined so that if $u$ obeys $h u=E u$, then $\Phi(n)=\left(\begin{array}{c}u(n+1) \\ u(n)\end{array}\right)$ obeys

$$
\Phi(n)=T(E, n, m) \Phi(m) .
$$

Constancy of the Wronskian implies $\operatorname{det} T=1$ so $\left\|T^{-1}\right\|=\|T\|$ and thus by (2.1)

$$
C(E)=\sup _{n, m}\|T(E, n, m)\| \leq \sup _{n}\|T(E, n, 0)\|^{2}
$$

is finite if and only if $E \in S$. We'll prove Theorem 2 in the following explicit form:

Theorem 2J. If $E \in S$, then

$$
\begin{aligned}
\underline{\lim } \operatorname{Im} m_{+}(E+i \epsilon) & \geq \frac{1}{4} C^{-3}, \\
\varlimsup & m_{+}(E+i \epsilon) \mid \leq 4 C^{3},
\end{aligned}
$$

where $C(E)$ is given by $(2.2)$. 
Proof. Let

$$
A(E, n) \equiv\left(\begin{array}{cc}
E-V(n) & -1 \\
1 & 0
\end{array}\right)
$$

so $T(E, n, 0)=A(E, n) T(E, n-1,0)$. It follows (as a telescoping sum) that

$$
T(E+i \epsilon, n, 0)=T(E, n, 0)+\sum_{j=0}^{n-1}(i \epsilon) T(E, n, j+1)\left(\begin{array}{ll}
1 & 0 \\
0 & 0
\end{array}\right) T(E+i \epsilon, j, 0)
$$

so by iteration, we get

$$
\|T(E+i \epsilon, n, 0)\| \leq \sum_{k=0}^{n}\left(\begin{array}{l}
n \\
k
\end{array}\right) C^{k+1} \epsilon^{k}=C(1+C \epsilon)^{n} \leq C e^{\epsilon C n} .
$$

By $\left\|T^{-1}\right\|=\|T\|$, we see that

$$
\left\|\left(\begin{array}{c}
u_{+}(E+i \epsilon, n+1) \\
u_{+}(E+i \epsilon, n)
\end{array}\right)\right\| \geq C^{-1} e^{-\epsilon C n}\left(\left|m_{+}(E+i \epsilon)\right|^{2}+1\right)^{1 / 2}
$$

since $\left(\begin{array}{l}u_{+}(E+i \epsilon, 1) \\ u_{+}(E+i \epsilon, 0)\end{array}\right)=\left(\begin{array}{c}m_{+}(E+i \epsilon) \\ 1\end{array}\right)$.

Squaring and summing over $n=1,3, \ldots$ we see that

$$
\sum_{n=1}^{\infty}\left|u_{+}(E+i \epsilon, n)\right|^{2} \geq C^{-2} e^{-2 \epsilon C}\left(1-e^{-4 \epsilon C}\right)^{-1}\left(\left|m_{+}(E+i \epsilon)\right|^{2}+1\right) .
$$

Thus by (1.7)

$$
\operatorname{Im} m_{+}(E+i \epsilon) \geq \frac{1}{4} C^{-3} e^{-2 \epsilon C}(4 \epsilon C)\left(1-e^{-4 \epsilon C}\right)^{-1}\left[1+\left|m_{+}(E+i \epsilon)\right|^{2}\right]
$$

or

$$
\underline{\lim }\left[\operatorname{Im} m_{+}(E+i \epsilon) /\left[1+\left|m_{+}(E+i \epsilon)\right|^{2}\right]\right] \geq \frac{1}{4} C^{-3} .
$$

Noting that $\left(1+\left|m_{+}\right|^{2}\right)^{-1} \leq 1$, we see that (2.6) immediately implies (2.3). And since $\left(1+\left|m_{+}\right|^{2}\right) / \operatorname{Im} m_{+} \geq\left|m_{+}\right|$, it also implies (2.4).

With only minor changes, the theorem extends to the general Jacobi matrix (tridiagonal self-adjoint) matrix:

$$
(h u)(n)=a_{n+1} u(n+1)+a_{n} u(n-1)+b_{n} u(n)
$$

so long as there is $\alpha$ finite with

$$
\alpha^{-1}<\left|a_{n}\right|<\alpha
$$

for all $n$. If $d \rho$ is the spectral measure for $u(n)=\delta_{1 n}$, then

$$
\int \frac{d \rho(E)}{z-E}=m_{+}(z)
$$

where $m_{+}(z)$ is defined to be $a_{1}^{-1} u_{+}(1)$ (if $u_{+}$is normalized by $u_{+}(0)=1$ ). (1.7) becomes

$$
\operatorname{Im} m_{+}(E)=a_{1}^{-2}(\operatorname{Im} E) \sum_{n=1}^{\infty}\left|u_{+}(n, E)\right|^{2} .
$$


It is no longer true that $\left\|T(E, n, 0)^{-1}\right\|=\|T(E, n, 0)\|$ since $\operatorname{det}(T(E, n, 0))$ may not be 1. Rather $\operatorname{det}(T(E, n, 0))=\frac{a_{1}}{a_{n}+1}$ so using (2.8), (2.2) becomes $C(E) \leq$ $\alpha^{2} \sup \|T(E, n, 0)\|^{2}$. (2.5) becomes

$$
\|T(E+i \epsilon, n, 0)\| \leq C(1+C \alpha \epsilon)^{n} \leq C e^{\epsilon C n \alpha}
$$

and (2.6) becomes

$$
\underline{\varliminf}\left[\operatorname{Im} m_{+}(E+i \epsilon)\right] /\left[1+a_{1}^{2}\left|m_{+}(E+i \epsilon)\right|^{2}\right] \geq \frac{1}{4} a_{1}^{-2} C^{-3} \alpha^{-1} .
$$

\section{§3. ThE SCHrÖDINGER CASE}

To carry the proof through from the discrete case, we must use (1.3) to bound $u^{\prime}$ locally by $u$. This is a standard Sobolev-type estimate; we haven't tried to optimize constants.

Lemma 3.1. If $u$ obeys $-u^{\prime \prime}+V u=E u$, then

$$
\left|u^{\prime}(x)\right|^{2} \leq\left[4+\frac{3}{4} \Gamma(|V-E|)\right] \int_{x-1}^{x+1}|u(y)|^{2} d y
$$

where $\Gamma$ is given by (1.3).

Proof. By Taylor's theorem with remainder,

$$
f^{\prime}(0)=\frac{1}{2} \frac{f(x)-f(-x)}{x}-\frac{1}{2 x} \int_{0}^{x}(x-y)\left[f^{\prime \prime}(y)+f^{\prime \prime}(-y)\right] d y .
$$

Integrate this from $\frac{1}{2}$ to 1 to get

$$
\left|f^{\prime}(0)\right| \leq \int_{-1}^{1}|f(x)| d x+\frac{3}{8} \int_{-1}^{1}\left|f^{\prime \prime}(x)\right| d x
$$

Let $f(y)=u(y+x)$ and use $u^{\prime \prime}=(V-E) u$ and the Schwarz inequality to get (3.1).

By (3.1), if $E \in S, u^{\prime}$ is also bounded and thus the transfer matrix $T(E, x, y)$ defined by

$$
T(E, x, y)\left(\begin{array}{l}
u^{\prime}(y) \\
u(y)
\end{array}\right)=\left(\begin{array}{l}
u^{\prime}(x) \\
u(x)
\end{array}\right)
$$

is bounded. Let

$$
C(E) \equiv \sup _{x, y}\|T(E, x, y)\| .
$$

Theorem 2S. Let $E \in S$ and define $A(E)=\frac{1}{2} C(E)^{-3} /\left(9+\frac{3}{2} \Gamma(|E-V|)\right)$. Then

$$
\underline{\lim } \operatorname{Im} m_{+}(E+i \epsilon) \geq A
$$

$$
\varlimsup\left|m_{+}(E+i \epsilon)\right| \leq A^{-1} \text {. }
$$


Proof. By mimicking the proof of (2.5), using integrals in place of sums,

$$
\|T(E+i \epsilon, x, 0)\| \leq C e^{\epsilon C|x|} .
$$

By (3.1)

$$
\int_{1}^{\infty}\left|u^{\prime}(x)\right|^{2} d x \leq\left[8+\frac{3}{2} \Gamma(|V-E-i \epsilon|)\right] \int_{0}^{\infty}|u(y)|^{2} d y
$$

so if $\beta=1 /\left(9+\frac{3}{2} \Gamma\right)$, then

$$
\begin{aligned}
\int_{0}^{\infty}|u(y)|^{2} d y & \geq \beta \int_{1}^{\infty}\left[|u(x)|^{2}+\left|u^{\prime}(x)\right|^{2}\right] d x \\
& \geq C^{-2} \beta\left(1+\left|m_{+}\right|^{2}\right) \int_{1}^{\infty} e^{-2 \epsilon C x} d x
\end{aligned}
$$

so by (1.8),

$$
\operatorname{Im} m_{+} \geq \frac{1}{2} C^{-3} \beta e^{-\epsilon C}\left(1+\left|m_{+}\right|^{2}\right)
$$

and the result follows as in the discrete case.

\section{Appendix 1: A Discrete Version of Weidmann's theorem}

One of the more interesting applications of Theorem 2 is the result of Weidmann [18], [19], [20] that if $V=V_{1}+V_{2}$, where $V_{1} \in L^{1}$ and $V_{2}$ is of bounded variation with $V_{2}(x) \rightarrow 0$ at infinity, then $-\frac{d^{2}}{d x^{2}}+V(x)$ has purely a.c. spectrum on $(0, \infty)$. A key to his argument is a proof that for any $E>0$, solutions are bounded. He does this by noting one can suppose $V_{2}$ is $C^{1}$ with $V_{2}^{\prime} \in L^{1}$ (by adjusting the breakup) and that if $K(x)=\left(u^{\prime}\right)^{2}+\left(E-V_{2}\right) u^{2}$, then $K^{\prime}(x)=2 V_{1} u^{\prime} u-2 V_{2}^{\prime} u^{2} \leq C\left(\left|V_{1}\right|+\left|V_{2}^{\prime}\right|\right) K(x)$ for $x$ large. Here we'll prove a result of Máté and Nevai [13] using a discrete analog of Weidmann's approach:

Theorem A.1. Let $v_{n}$ be a sequence on $\{1,2, \ldots\}$ so that $v_{n} \rightarrow 0$ and

$$
\sum_{n=1}^{\infty}\left|v_{n+1}-v_{n}\right|<\infty
$$

Then, the operator $h$ of (1.1) has purely absolutely continuous spectrum on $(-2,2)$.

Remarks. 1. (A.1) implies $\lim v_{n}$ exists so by adding a constant, it is no loss to suppose $v_{n} \rightarrow 0$.

2. If $v_{n} \in \ell^{1}$, then (A.1) holds so we don't need to consider sums as Weidmann does in the continuous case.

Proof. Given a solution of $h u=E u$, let

$$
K_{n}=u_{n+1}^{2}+u_{n}^{2}+\left(v_{n}-E\right) u_{n} u_{n+1} .
$$

Then

$$
\left(K_{n+1}-K_{n}\right)=\left(u_{n+2}-u_{n}\right)\left(u_{n+2}+u_{n}+\left(v_{n+1}-E\right) u_{n+1}\right)+\left(v_{n}-v_{n+1}\right) u_{n} u_{n+1}
$$


SO

$$
\left|K_{n+1}-K_{n}\right| \leq\left|v_{n}-v_{n+1}\right|\left|u_{n} u_{n+1}\right| .
$$

Suppose now $E \in(-2,2)$. Then for $n \geq$ some $N_{0}, 2-\left|v_{n}-E\right| \geq \delta>0$. For such $n$,

$$
K_{n} \geq \frac{\delta}{2}\left(u_{n+1}{ }^{2}+{u_{n}}^{2}\right)+\left(1-\frac{\delta}{2}\right)\left(\left|u_{n+1}\right|-\left|u_{n}\right|\right)^{2} \geq \frac{\delta}{2}\left(u_{n+1}{ }^{2}+u_{n}{ }^{2}\right)
$$

so (A.2) becomes

$$
K_{n+1} \leq\left(1+\frac{2}{\delta}\left|v_{n}-v_{n+1}\right|\right) K_{n}
$$

and for all $n \geq N_{0}$ :

$$
K_{n} \leq \prod_{m=N_{0}}^{\infty}\left(1+\frac{2}{\delta}\left|v_{m}-v_{m+1}\right|\right) K_{N_{0}} .
$$

The product is convergent by (A.1).

By using the remark at the end of Section 1, Theorem A.1 extends to the operator (2.7) so long as (2.8) holds and

$$
\begin{array}{ll}
b_{n} \rightarrow 0, & \sum_{n=1}^{\infty}\left|b_{n+1}-b_{n}\right|<\infty, \\
a_{n} \rightarrow 1, & \sum_{n=1}^{\infty}\left|a_{n+1}-a_{n}\right|<\infty .
\end{array}
$$

We merely define $K_{n}$ by

$$
K_{n}=a_{n+1} u_{n+1}^{2}+a_{n} u_{n}^{2}+\left(b_{n}-E\right) e_{n} u_{n+1} .
$$

This is related to results of [6].

\section{ApPendix 2: Eigenfunctions for Weidmann's theOREM}

We want to further elucidate Weidmann's theorem by showing how to actually find the asymptotics of the eigenfunctions. We'll suppose $V(x)=V_{1}(x)+V_{2}(x)$ with $V_{1} \in L^{1}$ and $V_{2}$ a $C^{1}$ function with $V_{2}^{\prime} \in L^{1}$ and $V_{2} \rightarrow 0$ at infinity. We claim:

Theorem B.1. Fix $E=k^{2}>0$ with $k>0$. Then every solution of

$$
\left(-\frac{d^{2}}{d x^{2}}+V(x)\right) u=E u
$$

is bounded; indeed, there exist $a, b$ so that

$$
\begin{aligned}
\left|u(x)-a u_{+}(x)-b u(x)\right| & \rightarrow 0, \\
\left|u^{\prime}(x)-i a k u_{+}(x)+i b k u_{-}(x)\right| & \rightarrow 0,
\end{aligned}
$$

where

$$
u_{ \pm}(x)=\exp \left( \pm i \int_{x_{0}}^{x} \sqrt{k^{2}-V_{2}(x)} d x\right)
$$

and $x_{0}$ is chosen so large that $V_{2}(x)<k^{2}$ for $x>x_{0}$. 
Remarks. 1. Since $\left(k^{2}-V_{2}(x)\right)^{-1 / 4} \rightarrow k^{-1 / 2}$, we could use the WKB form instead of (B.1), but the form (B.1) is what enters naturally.

2. This theorem and proof can be regarded as specializations of arguments in Hinton-Shaw [10].

Proof. Define $u_{ \pm}$by (B.1). Note that $u_{ \pm}$are $C^{2}$ and

$$
-u_{ \pm}^{\prime \prime}+(V(x)-E) u_{ \pm}=F_{ \pm} u_{ \pm}
$$

where

$$
F_{ \pm}(x)=V_{1}(x) \pm \frac{i}{2} V_{2}^{\prime}(x)\left(k^{2}-V_{2}(x)\right)^{-1 / 2}
$$

is in $L^{1}$ near infinity.

Let $W(x)$ be the Wronskian of $u_{+}$and $u_{-}$. Clearly, $W(x)=2 i k+o(1)$. Define $a(x), b(x)$ by the equations (variation of parameters)

$$
\begin{aligned}
u(x) & =a(x) u_{+}(x)+b(x) u_{-}(x), \\
u^{\prime}(x) & =a(x) u_{+}^{\prime}(x)+b(x) u_{-}^{\prime}(x) .
\end{aligned}
$$

A straightforward and standard calculation (see prob. 98 on pg. 395 of [15]) shows that $a, b$ obey the equations

$$
\left(\begin{array}{l}
a(x) \\
b(x)
\end{array}\right)^{\prime}=M(x)\left(\begin{array}{l}
a(x) \\
b(x)
\end{array}\right)
$$

where

$$
M(x)=W(x)^{-1}\left(\begin{array}{cc}
-F_{+} & -u_{-}^{2} F_{-} \\
F_{+} u_{+}^{2} & F_{-}
\end{array}\right) .
$$

Since this is in $L^{1}$, standard arguments show that $\lim _{k \rightarrow \infty}\left(\begin{array}{l}a(x) \\ b(x)\end{array}\right)=\left(\begin{array}{l}a \\ b\end{array}\right)$ exists.

If, moreover, $V$ obeys (1.3) (a mild restriction), this and Theorem 1 imply that $\sigma_{\mathrm{ac}}(H)=[0, \infty), \sigma_{\text {sing }} \cap(0, \infty)=\emptyset$.

\section{NOTE ADDED IN PROOF}

Subsequent to our work, S. Jitomirskaya and Y. Last [Phys. Rev. Lett. 76 (1996), 1765-1769 and papers in preparation] have shown how to get explicit $m$ function bounds in the general situation covered by Gilbert-Pearson theory.

\section{REFERENCES}

[1] N. Aronszajn, On a problem of Weyl in the theory of Sturm-Liouville equations, Am. J. Math. 79 (1957), 597-610. MR 19:550

[2] H. Behncke, Absolute continuity of Hamiltonians with von Neumann Wigner potentials, II, Manu-scripta Math. 71 (1991), 163-181. MR 93f:81031

[3] P. Briet and E. Mourre, Some resolvent estimates for Sturm-Liouville operators, preprint.

[4] R. Carmona, One-dimensional Schrödinger operators with random or deterministic potentials: New spectral types, J. Funct. Anal. 51 (1983), 229-258. MR 85k:34144

[5] E. Coddington and N. Levinson, Theory of Ordinary Differential Equations, McGraw-Hill, New York, 1955. MR 16:1022

[6] J. Dombrowksi and P. Nevai, Orthogonal polynomials, measures, and recurrence relations, SIAM J. Math. Anal. 17 (1986), 752-759. MR 87g:42039

[7] W. Donoghue, On the perturbation of spectra, Commun. Pure Appl. Math. 18 (1965), 559579. MR 32:8171

[8] D.J. Gilbert, On subordinacy and analysis of the spectrum of Schrödinger operators with two singular endpoints, Proc. Roy. Soc. Edinburgh Sect. A 112 (1989), 213-229. MR 90j:47061 
[9] D.J. Gilbert and D.B. Pearson, On subordinacy and analysis of the spectrum of onedimensional Schrödinger operators, J. Math. Anal. Appl. 128 (1987), 30-56. MR 89a:34033

[10] D.B. Hinton and J.K. Shaw, Absolutely continuous spectra of second order differential operators with short and long range potentials, Siam J. Math. Anal. 17 (1986), 182-196. MR 87c:34038

[11] S. Khan and D.B. Pearson, Subordinacy and spectral theory for infinite matrices, Helv. Phys. Acta 65 (1992), 505-527. MR 94a:47066

[12] A. Kiselev, Absolutely continuous spectrum of one-dimensional Schrödinger operators and Jacobi matrices with slowly decreasing potentials, preprint.

[13] A. Máté and P. Nevai, Orthogonal polynomials and absolutely continuous measures, Approximation Theory IV (C.K. Chui, L.L. Schumaker, and J.D. Ward, eds.), Academic Press, New York, 1983. MR 85j:42044

[14] D.B. Pearson, Quantum Scattering and Spectral Theory. Techniques of Physics, vol. 9, Academic Press, London, 1988. MR 91k:81198

[15] M. Reed and B. Simon, Methods of Modern Mathematical Physics, III. Scattering Theory, Academic Press, New York, 1979. MR 80m:81085

[16] B. Simon, Spectral analysis of rank one perturbations and applications, Proc. Mathematical Quantum Theory II: Schrödinger Operators (J. Feldman, R. Froese, and L.M. Rosen, eds.), CRM Proc. Lecture Notes, vol. 8, Amer. Math. Soc., Providence, RI, 1995, pp. 109-149. CMP 95:12

[17] G. Stolz, Bounded solutions and absolute continuity of Sturm-Liouville operators, J. Math. Anal. Appl. 169 (1992), 210-228. MR 93f:34141

[18] J. Weidmann, Zur Spektraltheorie von Sturm-Liouville-Operatoren, Math. Z. 98 (1967), 268302. MR 35:4769

[19] - Absolut stetiges Spektrum bei Sturm-Liouville-Operatoren und Dirac-Systemen, Math. Z. 180 (1982), 423-427. MR 83m:34023

[20] _ Spectral Theory of Ordinary Differential Operators, Lecture Notes in Mathematics Vol. 1258, Springer-Verlag, Berlin/Heidelberg, 1987. MR 89b:47070

Division of Physics, Mathematics, and Astronomy, California Institute of Technology, 253-37, Pasadena, California 91125

E-mail address: bsimon@caltech.edu 